\title{
SESSION 3
}

\section{TECHNIQUES OF MAGNETIC FIELD MEASUREMENTS}




\title{
PROGRESS IN THE MEASUREMENT OF SOLAR MAGNETIC FIELDS
}

\section{GUOXIANG AI}

Beijing Astronomical Observatory, Chinese Academy of Sciences, Beijing 100080, People's Republic of China

\begin{abstract}
The historical development of optical instruments for solar physics is outlined, from white light to unpolarized and polarized monochromatic light, to Stokes profiles and simultaneous fields of view, from points to lines, plane to cube. An evolutionary series and classificaton of instruments for the solar magnetic field is described. As a next step the 2-D real time polarizing spectrograph has been proposed. The planned instruments in China for measurements of solar magnetic and velocity fields are briefly introduced.
\end{abstract}

\section{INTRODUCTION}

Eight years ago J.W. Harvey (1985) contributed a very complete and excellent review on "trends in measurement of solar vector magnetic fields using the Zeeman effect". The main conclusions of the review are still correct and relevant today. In the present paper, two aspects will be discussed to supplement Harvey's review. Firstly we give an overview of the history of the development of optical instruments for solar physics. Secondly we discuss the next type of instrument, a two-dimension real time polarizing spectrograph. Some proposals and plans for measurements of solar magnetic and velocity fields in China will also be introduced briefly.

\section{BRIEF OVERVIEW OF THE HISTORY OF SOLAR OPTICAL INSTRUMENTS}

In 1611 the Galilean telescope opened the door to modern astronomy. The development of optical instruments for solar physics during the following about 400 years is shown in Fig. 1. The vertical axis characterizes the complexity of the radiation parameters analysed, from white light, spectrum or monochromatic light, polarized monochromatic light, to Stokes profiles. The horizontal axis indicates the spatial dimension and simultaneous field of view, from point, line, plane, to cube. From Fig. 1 it is clear that there is a logical relation between the advance of the complexity of the radiation parameter analysed and the improvements of the simultaneous field of view. The pioneering measurements of solar magnetic fields were done by G.E. Hale (1908). He invented the first generation of instruments to measure the solar magnetic field by the Zeeman effect in 1908 and observed the strong magnetic fields of sunspots. The second 


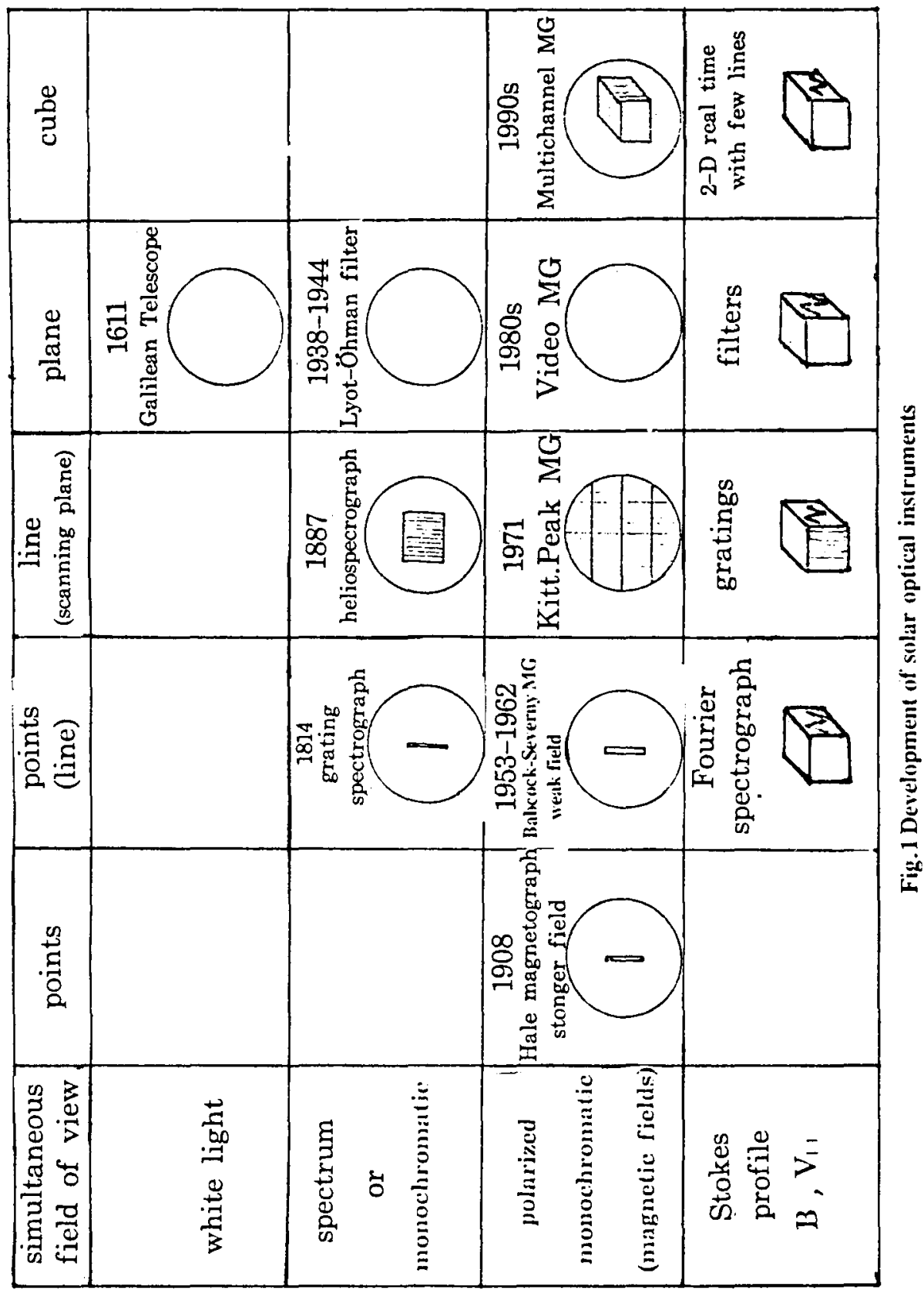


generation was also invented at the Mount Wilson Observatory in 1952 by H.W. Babcock (1953), who developed a photoelectric device to measure the weaker fields. Later a system to measure the vector magnetic field was developed at the Crimean Astrophysical Observatory (Severny, 1962), but all these systems could only record one spatial point at a time. In 1971 Livingston et al. (1971) developed the third generation instrument, which had a linear field of view through the use of a linear array with $512 \times 1$ pixels. This powerful instrument has been used to record daily full-disk magnetograms. The recording efficiency was increased 512 times as compared with the Babcock system, and it allowed higher spatial and temporal resolutions. The fourth generation instrument is the video magnetograph, in which a narrow-band birefringent filter replaces the traditional grating spectrograph, and a CCD camera at video rates is used. This system has been implemented in the 1980's (Hagyard et al. 1982; Zirin, 1986; Ai and $\mathrm{Hu}, 1986$ ). It has a very high recording efficiency with $512 \times 512$ or $1024 \times 1024$ pixels. In China the video magnetograph is called the Solar Magnetic Field Telescope, which was proposed in 1966 based on the considerations of Fig. 1, and which was completed in 1987 after 20 years of research and construction.

In the past decade the video magnetograph has become a major tool for investigations of solar magnetic fields. Its great power combining about $0.5^{\prime \prime}$ angular resolution with about one minute temporal resolution and high sensitivity has allowed many new results to be obtained, e.g. the fine structure of the magnetic field in active and quite regions, round the clock observations (at Big Bear and Huairou) to explore the long time evolution of the magnetic field, magnetic shear, etc. Because of its high quality and relatively low cost, the video system has become popular worldwide.

With the beginning of the $1990 \mathrm{~s}$, two developments have taken place. The first concerns the multi-channel video magnetograph. The multi-channel birefringent filter ( $\mathrm{Ai}$ and $\mathrm{Hu}, 1987 \mathrm{a}, \mathrm{b}, \mathrm{c}$ ) allows several spectral lines from different solar layers to be observed simultaneously to study the 3-D structure of the vector magnetic field and electrical field of the solar atmosphere. It may be called the fifth generation magnetograph due to it cubic field of view. The second concerns the investigation of Stokes profiles of magnetically sensitive lines. The main aim is to accurately determine the vector magnetic field. The collaboration between HAO and SPO (Lites et al. , 1991) and work at the University of Hawaii (Canfield et al., 1991) and MSFC (Gary et al., 1991) have brought good results, which are very useful for the calibration of the vector magnetic field, in particular for the umbra of sunspots.

\section{THE NEXT STEP}

In his review Harvey (1985) pointed out: "A difficult problem is to acquire polarization, spectral and spatial information, simultaneously (or at least rapidly) at a large number of points. No practical solution that avoids scanning in some domain seems to have been discovered." In my understanding, what Harvey has in mind is a two-dimensional real time (simultaneous) polarizing spectrograph.

During the past half century three types of imaging (2-D) polarizing spectrographs have been developed. The grating spectrograph can be used for 2$\mathrm{D}$ imaging by spatial scanning with the slit, the Fourier transform spectrometer 
(FTS) by scanning of the path difference, the universal birefringent filter by spectral scanning (cf. Fig. 2). Due to the scanning, simultaneity is lost. Three other quasi real-time 2-D spectrographs have been proposed, the subtractive double pass spectrograph (Mein, 1977), the multi-slit spectrograph, and the optical-fiber spectrograph. The first one does not get the continuous spectrum, the second one has no continuous spatial coverage, the third one also has no continuous spatial coverage and the total efficiency is not increased. Therefore Harvey (1985) concluded "no practical solution". A 2-D real time polarizing spectrograph based on the multi-channel filter however provides an effective method.

An instrument that can record the Stokes parameter line profiles over a simultaneous spatial field of view would provide a powerful tool for research on eruptive or rapidly varying processes in the 3-D solar atmosphere and corona, on magnetic turbulence, the interaction of magnetic and velocity fields, and so on. A variety of solar physics parameters would be provided, not only the magnetic and velocity fields, but also other parameters with high spatial and time resolutions as well as high accuracy.

\begin{tabular}{|c|c|c|}
\hline $\begin{array}{l}\text { Grating spectrograph } \\
\text { slit } \\
\text { spectrum }\end{array}$ & $\begin{array}{l}\text { Imaging spectrograph } \\
\text { 2-D } \\
\text { spatial scanning with slit }\end{array}$ & $\searrow$ \\
\hline & $\begin{array}{l}\text { Fourier spectrograph } \\
\text { scanning the path difference }\end{array}$ & $\begin{array}{l}\text { 2-D real time } \\
\text { spectrograph }\end{array}$ \\
\hline $\begin{array}{l}\text { Lyot-Ö hman filter } \\
\text { monochromatic image }\end{array}$ & $\begin{array}{l}\text { Universal birefringent filter } \\
\text { 2-D } \\
\text { spectral scanning }\end{array}$ & $\lambda$ \\
\hline
\end{tabular}

Fig. 2. Trends in instrumentation for spectral analysis.

\section{2-D REAL TIME SPECTROGRAPH}

The principle of the polarizing beam splitter PBS (Banning, 1947) is illustrated in Fig. 3a. Part of the incident light is reflected at the Brewster angle from a multi-layer film surface, while the rest is transmitted. The reflected (S) and the transmitted (P) beams have mutually orthogonal linear polarizations. The PBS has not yet been used in birefringent filters, because the polarizing degree of the PBS is slightly lower (99\%) than required for the filter. To overcome this, we show in Fig. 3b how two polaroids with a polarizing degree of about $90 \%$ are inserted immediately behind the PBS, which gives a polarization degree of the two emergent beams of up to about $99 \%$. The resulting degree of polarization then becomes $p=1-(1-0.99) \times \frac{1}{2}(1-0.90)=0.9995$. This value fully satisfies the need for the filter. Figure $4 \mathrm{a}$ illustrates an element of a Lyot filter. When the polarization direction of $\mathrm{P} 2$ is parallel to that of $\mathrm{P} 1(\mathrm{P} 2 \| \mathrm{P} 1)$, the transmission is $T_{\|}=\cos ^{2}(\pi \mu d / \lambda)$, where $\mu$ is the birefringent index, and $d$ is the thickness of the birefringent crystal. When $\mathrm{P} 2 \perp \mathrm{P} 1$, one gets $T_{\perp}=\sin ^{2}(\pi \mu d / \lambda)$. $T_{\|}$and $T_{\perp}$ 

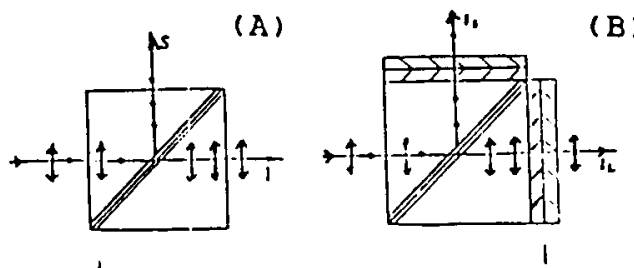

Fig.3 (a) PBS

(b) CPBS

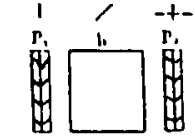

(A)

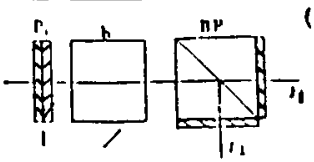

(B)

(b) MCBF element.

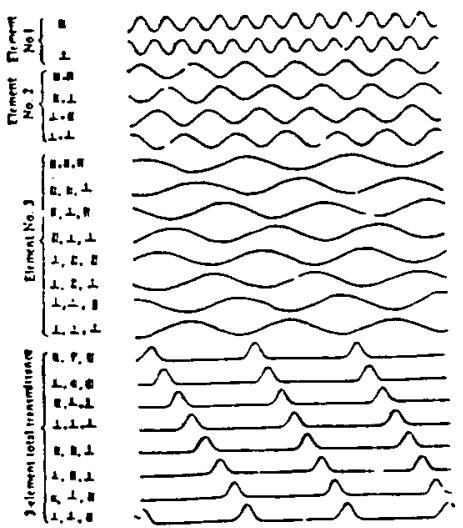

Fig.5 8-channel MCBF.

Fig.6 Transmission of 8-channel.

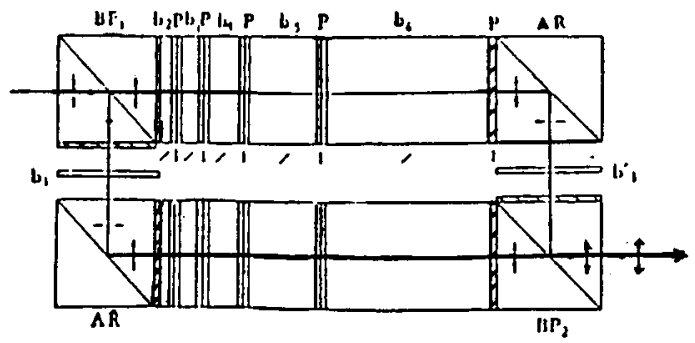

Fig.7 Scheme of TTBF for galactic and stellar observations. 
complement each other in the transmission spectrum, with $T_{\|}+T_{\perp} \equiv 1$. When the polaroids are used, only one beam and one channel can be obtained. The other beam is absorbed. When a CPBS replaces the polaroid as in Fig. $4 \mathrm{~b}$, two beams, $I_{\|}$and $I_{\perp}$, are available, and one has a 2 -channel element. When combinations of 2-channel elements are used in the filter, a multi-channel birefringent filter (MCBF) can be obtained. The quantitative relation between $M$, the number of channels, and $n$, the number of MCBF elements, is $M=2^{n}$. A polarizing solid Michelson interference filter (PSMIF) has been constructed by Title and Ramsey (1980). The spectral resolution could reach about $0.01 \AA$. A multichannel PSMIF can be constructed in a way similar to that of the MCBF.

The first example of MCBF is a 2-D real time spectrograph. When the thickest crystal is located at the beginning, and the thinner crystals are placed successively later along the light path, a 2-D spectrograph can be obtained. A 2-D spectrograph with 8 channels together with its spectral transmission curves are shown in Figs. 5 and 6 . The thickest crystal is common to all the channels, while the other crystals are generally common to several channels. The amount of crystal needed is therefore greatly reduced. The quantitative relation between $M$, the number of the channels, and $D$, the total crystal thickness in units of the thickness of a traditional birefringent filter with a single channel, is $M=4^{D-1}$.

If two identical birefringent filters are placed in the two beams emerging from the first CPBS, then the beams from these two filters represent those of totally transmitting birefringent filters (TTBF). This is so because the TTBF utilizes the half of the light that is absorbed by the first polaroid in a conventional filter. Its transmission is therefore twice that of a conventional filter. Using the same procedure for each of the split beams, one can obtain a multi-channel TTBF. A TTBF with a transmission of $60 \%$ is shown in Fig. 7 .

In the MCBF the broad channels transmitting the selected spectral lines are placed first, behind which the narrower elements are placed, separated in the different channels. The merits of a multi-channel filter with a multi-channel head are the following: (1) The thickest crystal must not be located at the beginning, which means that the heat load on it may be reduced. (2) Every channel may have an independent bandwidth suitable to the particular need. (3) Behind the multi-channel head one may place many Fabry-Perot and/or Daystar filters. (4) Filters which are available in an observatory can be combined with the multichannel head and used for simultaneous observations.

\section{SOME PROPOSALS AND PLANS IN CHINA}

(1) A series of new filters are under development:

A. 2-D real time polarizing spectrograph with 64 channels, for line profiles.

- Universal filter system for the range $3800-7000 \AA$.

- FWHM: $0.05 \AA$ at $6563 \AA, 0.015 \AA$ at $3933 \AA$.

- Main objective: Stokes line profiles from space and from ground.

- Can be accomodated in a $60-200 \mathrm{~cm}$ diameter optical solar telescope.

B. 2-D real time polarizing spectrograph with 8 channels, for one spectral line.

- Universal filter system for the range $4600-6600 \AA$.

- FWHM: $1 / 8 \AA$ at $\mathrm{H} \alpha$.

- Main objective: Stokes profiles of chromospheric lines. 
- Both continuously distributed or discretely positioned channels (the latter may be needed to accomodate observations of large Doppler velocities).

C. Totally transmitting birefringent filter.

- Universal filter system for the range 4600-6600 $\AA$.

- FWHM: $0.15 \AA$ at $5324 \AA$.

- Transmission about $30 \%$ (including an interference filter).

- Main objective: System for a space telescope with smaller entrance aperture; ground-based system for short exposure times, as needed for speckle interferometry.

D. $10830 \AA$ birefringent filter for He observations of flares and coronal holes with a FWHM of $0.5 \AA$.

(2) A group of about sixteen scientists and engineers will be set up for feasibility studies and the design of the following two telescopes:

- Space-based solar telescope with a 2-D spectrograph.

- A movable solar optical telescope with an entrance aperture of 60-65 cm and a totally transmitting filter to search for a site with excellent seeing in the west part of China. A seeing of $0.2^{\prime \prime}$ is aimed for.

(3) Collaboration between Chinese and foreign observatories, both in space and on ground, is anticipated.

\section{REFERENCES}

Ai G. and Hu Y. 1986, Publ. Beijing Astron. Obs., 8.

Ai G., Hu Y. 1987a, Science in China, p.868.

Ai G., Hu Y. 1987b, Science in China, p. 1969.

Ai G., Hu Y. 1987c, Acta Astrophysica Sinica, 7, 305.

Ai G. 1991, in L.J. November (ed.), Solar Polarimetry, NSO/Sacramento Peak, Sunspot, NM, p.96.

Babcock, H.W. 1953, Ap. J., 118, 387.

Banning, B. 1947, J. Opt. Soc. Am., 37, 729.

Canfield R.C., Yuhong Fan, Leka, K.D., McClymont, A.N., Wülser, J.-P. 1991, in L.J. November (ed.), Solar Polarimetry, NSO/SPO, p. 296.

Gary, G.A., Hagyard, M.J., West, E.A. 1991, in L.J. November (ed.), Solar Polarimetry, NSO/Sacramento Peak, Sunspot, NM, p. 65.

Hagyard, M.J., Cumings, N.P., West, E.A., Smith, J.E. 1982, Solar Phys., 80, 33.

Hale, G.E. 1908, Ap. J., 28, 315.

Harvey, J. 1985, in M.J. Hagyard (ed.), Measurements of Solar Vector Magnetic

Fields, NASA Conf. Publ. 2374, p. 109.

Livingston, W.etal. 1971, Publ. Roy. Obs. Edinburgh, 8, 52.

Lites, B.W., Elmore, D., Murphy, G., Skumanich, A., Tomczyk, S. 1991, 1991 in L.J. November (ed.), Solar Polarimetry, NSO/SPO, p. 3.

Mein, P. 1977, Solar Phys., 54, 45.

Severny, A.B. 1962, Trans. IAU, IIB, 426.

Title, A., Ramsey, H. 1980, Appl. Optics, 19, 2046.

Zirin, H. 1986, Austr. J. Phys., 38, 961. 